\title{
Serum Metabolomics Reveals Cholic Acid, Chenodeoxycholic Acid, and Taurochenodeoxycholic Acid as Potential Biomarkers for Hypothyroid Rats
}

\author{
Hidenori Nagao $^{1}{ }^{*}$, Masanori Suzuki ${ }^{2}$, Hironori Aoki ${ }^{1}$, Kouichi Minato ${ }^{1}$ \\ ${ }^{1}$ Pharmacokinetics Research Department of ASKA Pharmaceutical Co., Ltd., Kawasaki, Japan \\ ${ }^{2}$ Department of Analytical Research, ASKA Pharma Medical Co., Ltd., Kawasaki, Japan
}

Email address:

nagao-h@aska-pharma.co.jp (H. Nagao)

\section{To cite this article:}

Hidenori Nagao, Masanori Suzuki, Hironori Aoki, Kouichi Minato. Serum Metabolomics Reveals Cholic Acid, Chenodeoxycholic Acid, and Taurochenodeoxycholic Acid as Potential Biomarkers for Hypothyroid Rats. American Journal of Life Sciences.

Vol. 3, No. 4, 2015, pp. 295-305. doi: 10.11648/j.ajls.20150304.17

\begin{abstract}
Hypothyroidism decreases energy metabolism including carbohydrate and lipid metabolism and protein synthesis, due to reduced serum levels of the thyroid hormones thyroxine $\left(T_{4}\right)$ and triiodothyronine $\left(T_{3}\right)$. Although many endogenous serum metabolites are influenced by hypothyroidism, serum metabolomic profiling has rarely been applied to the study of hypothyroidism. In the present study, we investigated potential biomarkers for hypothyroidism using serum metabolomics, and then measured serum levels of these endogenous metabolites using an analytical method: ultra-performance liquid chromatography coupled with quadrupole time-of-flight mass spectrometry. There was a significant difference in the metabolic profiles of thyroidectomized (Tx) and normal rats. We found that many bile acid (BA) levels were significantly changed in serum of Tx rats. Simultaneous measurement of 12 different BAs in serum revealed that cholic acid (CA), chenodeoxycholic acid (CDCA), and taurochenodeoxycholic acid (TCDCA) levels significantly increased in Tx rats by approximately 25 -fold, 11 -fold, and 3-fold, respectively, compared with those of control rats. In $T x$ rats with active hormone $T_{3}$ replacement, serum $\mathrm{T}_{3}$ levels were returned to physiological levels. However, these changes in BA levels were maintained at a high level. These results indicate that $\mathrm{T}_{3}$ replacement does not normalize the thyroid hormonal milieu. Thus, increased CA, CDCA, and TCDCA levels in serum after $\mathrm{Tx}$ may be a homeostatic response to not only $\mathrm{T}_{3}$ but also pro-thyroid hormone $\mathrm{T}_{4}$ deficiency. This study is the first to report that CA, CDCA, and TCDCA may be potential biomarkers for hypothyroidism and the efficacy of thyroid hormone replacement therapy in hypothyroidism.
\end{abstract}

Keywords: Metabolomics, Biomarker, Bile Acid, Hypothyroidism, Thyroid Hormone Replacement Therapy

\section{Introduction}

Thyroid hormones (THs) play an important role in both energy homeostasis and regulation and also during the development and differentiation of cells. THs secreted from the thyroid gland are thyroxine $\left(\mathrm{T}_{4}\right)$ and triiodothyronine $\left(T_{3}\right) . T_{4}$ has little biological activity and is considered a pro-hormone, as activation occurs through deiodination of $\mathrm{T}_{4}$ at the 5'-position of the phenolic ring to form $\mathrm{T}_{3}$, the active form of thyroid hormone [1]. Most of the actions of $\mathrm{TH}$ seem to be dependent on binding of $\mathrm{T}_{3}$ to a nuclear thyroid hormone receptor (TR). There are three major forms of the TR, designated $\alpha 1, \beta 1$, and $\beta 2$. TR $\alpha 1$ is the predominant subtype in bone, the gastrointestinal tract, cardiac and skeletal muscle, and the central nervous system;
TR $\beta 1$ is most abundant in the liver and kidney; and TR $\beta 2$ is more discretely expressed in the hypothalamus, pituitary, cochlea, and retina [2-5]. Thus, THs act on a wide variety of tissues, and hypothyroidism results in multiple physiological responses. Some studies have shown that TH deficiency due to hypothyroidism leads to a decreased rate of metabolism of lipids, proteins, and carbohydrates [6,7].

Metabolomics, which can be defined as measurement of all cellular metabolites, is the comprehensive assessment of endogenous metabolites and quantification of metabolites from a biological sample [8]. In contrast to genomics, transcriptomics, and proteomics, which involve macromolecules with similar chemical properties, such as 
DNA, RNA, and proteins, metabolomics analysis deals with diverse properties of low molecular weight compounds. This technique can be widely applied to a number of fields including pharmacology, toxicology, and food science $[9,10]$. As metabolomics involves the downstream products of genomics and proteomics, it is also regarded as a complement to other "omics" for interpretation of gene function [11,12]. Metabolomics is a powerful tool for discovering new small molecule biomarkers. Following further validation, the identified markers can help to diagnose and evaluate prognosis for various diseases, such as diabetes mellitus, liver injury, and acute renal toxicity [13-15].

In this study, we used serum metabolomics profiling in rats with hypothyroidism induced by surgical thyroidectomy using ultra-performance liquid chromatography coupled with quadrupole time-of-flight mass spectrometry (UPLC-QTOF/MS). First, we analyzed the metabolites in serum, and detected differences in bile acids (BAs). These metabolites were analyzed using supervised orthogonal partial least squared discriminant analysis (OPLS-DA) and online metabolite data bases search. Then, concentrations of the detected BAs in serum were quantified using a simultaneous analytical method [16]. We investigated sequential changes in individual serum BA levels in rats after thyroidectomy, and then investigated the effects of replacement of the active hormone $\mathrm{T}_{3}$ on individual serum BA levels. Our results suggest that cholic acid (CA), chenodeoxycholic acid (CDCA), and taurochenodeoxycholic acid (TCDCA) may be potential biomarkers for hypothyroidism and the efficacy of $\mathrm{TH}$ replacement therapy in hypothyroidism.

\section{Materials and Methods}

\subsection{Materials}

$\mathrm{T}_{3}$ was purchased from Sigma-Aldrich Co. (St. Louis, MO, USA). CA and CDCA were purchased from Wako Pure Chemical Industries, Ltd. (Osaka, Japan). Glycocholic acid (GCA), tauroursodeoxycholic acid (TUDCA), TCDCA, and taurodeoxycholic acid (TDCA) were purchased from Merck (Darmstadt, Germany). Taurocholic acid (TCA), taurine- $\beta$-muricholic acid ( $\beta$ TMCA), glycochenodeoxycholic acid (GCDCA), taurolithocholic acid (TLCA), taurohyodeoxycholic acid (THDCA), and taurohyocholic acid (THCA) were purchased from Steraloids UK Ltd. (London, England). The internal standards, CA-2,2,3,4,4- $\mathrm{d}_{5}$ and TCA-2,2,4,4- $\mathrm{d}_{4}$, were purchased from Toronto Research Chemicals Inc. (Tronto, Canada), and GCA-2,2,4,4- $\mathrm{d}_{4}$ was from $\mathrm{C} / \mathrm{D} / \mathrm{N}$ isotopes Inc. (Quebec, Canada). Deionized water used for UPLC mobile phase was prepared on Milli-Q advantage A10 water purification system (Millipore, Bedford, MA, USA). Methanol and acetonitrile were of HPLC grade and obtained from Kanto Chemical Inc. (Tokyo, Japan) and Wako Pure Chemical Industries, Ltd. (Osaka, Japan), respectively. Formic acid and ammonium acetate were from Sigma Chemical (St. Louis, MO, USA).

\subsection{Animals}

Seven-week-old male Sprague-Dawley rats were obtained from Charles River Laboratories Japan (Kanagawa, Japan). Animals were fed a commercial diet (FR-2, Funabasi Farm Co., Chiba, Japan) and filtered tap water ad libitum. The cages were located in a light $(0800-2000 \mathrm{~h}$ lights on $)$, temperature $\left(23 \pm 5^{\circ} \mathrm{C}\right)$, and humidity $(60 \pm 20 \%)$ controlled room. The rats were allowed to acclimatize for 1 week before starting the experiments.

All experimental procedures were approved by the Animal Research Committee of ASKA Pharmaceutical Co., in accordance with the Basic Guidelines for Proper Conduct of Animal Testing and Related Activities in the Research Institutions under the Jurisdiction of the Ministry of Health, Labour and Welfare of Japan.

\subsection{Thyroidectomy}

Rats were made hypothyroid by surgical thyroidectomy as described previously in detail [17]. After the surgery, serum thyroid stimulating hormone (TSH) rapidly increased, and body weight gain achieved complete stasis. Complete resection of the thyroid in the thyroidectomized (Tx) rats was confirmed at the end of the experiment by macroscopic observation at necropsy. The Tx rats drank $1 \%$ calcium lactate aqueous solution ad libitum for 1 week after Tx. Normal group received sham operation as control.

\subsection{Experiment 1}

Rats were divided into two groups $(\mathrm{n}=5)$, the Tx and normal (control) groups. Blood were collected from tail vein for 3 weeks. All blood samples were obtained between 0900 and $1100 \mathrm{~h}$ to minimize fluctuations in THs [18]. Serum samples were separated by centrifugation at $16000 \times \mathrm{g}$ for $5 \mathrm{~min}$ at $4^{\circ} \mathrm{C}$, and stored at $-20^{\circ} \mathrm{C}$ until analyzed.

\subsection{Experiment 2}

Osmotic minipumps (Alzet, model 2ML4, DURECT, CA, USA), delivering $1.5 \mu \mathrm{g} \mathrm{T}_{3} /$ head/day $(\mathrm{Tx}+\mathrm{T} 3$ group; $\mathrm{n}=6$ ) were implanted subcutaneously in rats on day 0 under isoflurane anesthesia at 2 weeks after Tx. The doses of $\mathrm{T}_{3}$ were determined by our preliminary experiment and previous literature [19]. The vehicle was saline, in which $\mathrm{T}_{3}$ (final concentration; $25 \mu \mathrm{g} / \mathrm{mL}$ in $0.5 \mathrm{mmol} / \mathrm{L} \mathrm{NaOH} / \mathrm{saline}$ ) was diluted after $\mathrm{NaOH}$ solubilization $(5 \mathrm{mg} \mathrm{T} / 20 \mu \mathrm{L}$ of 1 $\mathrm{mol} / \mathrm{L} \mathrm{NaOH}$ ). Six normal and Tx rats (control and Tx group) received pumps with $0.5 \mathrm{mmol} / \mathrm{L} \mathrm{NaOH} / \mathrm{saline}$ only. On the day before pump implantation (day -1) and after implantation (day 14), blood were collected from tail vein between 0900 and $1100 \mathrm{~h}$. Serum samples were separated by centrifugation at $16000 \times \mathrm{g}$ for $5 \mathrm{~min}$ at $4^{\circ} \mathrm{C}$, and stored at $-20^{\circ} \mathrm{C}$ until analyzed. 


\subsection{Metabolomics Analysis}

\subsubsection{UPLC-QTOF/MS}

Serum samples which were collected on the final day (3 weeks after Tx) of the experiment 1, were prepared for UPLC-QTOF/MS analysis by acetonitrile protein precipitation. The serum samples $(40 \mu \mathrm{L})$ were mixed with cold acetonitrile $(80 \mu \mathrm{L})$ for $30 \mathrm{sec}$ and centrifuged at 16000 $\times \mathrm{g}$ for $5 \mathrm{~min}$ at $10^{\circ} \mathrm{C}$. An aliquot of the supernatant transferred to a clean tube. An equivalent $0.1 \%$ formic acid was added to the supernatant. Metabolite extracts $(2 \mu \mathrm{L})$ were injected onto a reverse-phase ACQUITY UPLC HSS T3 column $(2.1 \times 100 \mathrm{~mm}$ i.d., $1.8 \mu \mathrm{m}$ particles, Waters, Milford, MA, USA ) using an ACQUITY UPLC system (Waters). The flow rate of the mobile phase was $400 \mu \mathrm{L} / \mathrm{min}$. Mobile phase A consisted of $0.1 \%$ formic acid in water, while mobile phase $\mathrm{B}$ was acetonitrile. The gradient was initiated with $99 \%$ A and maintained for $2 \mathrm{~min}$, and then linearly decreased to $5 \%$ within $3 \mathrm{~min}$, and maintained for 4 min. The column eluent was introduced directly into the mass spectrometer. Mass spectrometry was performed on a Q-TOF instrument (Xevo QTof MS, Waters), operating in negative electrospray ionization ( $\mathrm{ESI}^{-}$) mode from $\mathrm{m} / \mathrm{z} 50$ to 1000 in MS scanning. Capillary voltage was $2.5 \mathrm{kV}$, sample cone was $25 \mathrm{~V}$, desolvation temperature $500^{\circ} \mathrm{C}$, source temperature $150^{\circ} \mathrm{C}$, and desolvation gas flow $1000 \mathrm{~L} / \mathrm{h}$. Leucine enkephalin $(\mathrm{m} / \mathrm{z}$ 556.2771) was used as the lock-mass; a solution of $0.2 \mathrm{ng} / \mathrm{mL}$ (50:50 acetonitrile: water containing $0.1 \%$ formic acid) was infused into the instrument at $5 \mu \mathrm{L} / \mathrm{min}$ via as auxiliary sprayer. MassLynx software (v.4.1, Waters) was used to acquire the chromatogram and mass spectrometric data in centroid format. In the $\mathrm{MS}^{\mathrm{E}}$ (Waters) was performed on all samples, where data were collected at both low $(5.0 \mathrm{eV})$ and high $(20.0$ to $35.0 \mathrm{eV})$ collision energies, in order to obtain fragmentation data simultaneously.

\subsubsection{Data Processing and Analysis}

The raw data of chromatographic peaks obtained from the UPLC-TOF/MS system were recognized and matched by MakerLynx XS software (Waters). A list of the detected peaks intensities was generated for the first chromatogram, and the retention time and $\mathrm{m} / \mathrm{z}$ value pairs were used as identifiers. The ion intensities for each detected peak were then normalized to the sum of the peak intensities in that sample. There was no significant correlation between the total intensities used for normalization and the sample groups being compared in the study. The multivariate data matrix was analyzed by EZ-info software (Waters). The supervised orthogonal partial least squares discriminant analysis (OPLS-DA) was used to concentrate group discrimination into the first component with remaining unrelated variation contained in subsequent components. Candidate markers were extracted from S-plots constructed following analysis with OPLS-DA, and markers were chosen based on their contribution to the variation and correlation within data set.

\subsubsection{Identification of Candidate Markers}

The elemental compositions were generated by MarkerLynx based on the accurate mass of the high-contribution-score metabolites. MassFragment application manager (Waters) was used to facilitate the MS/MS fragment ion analysis. The markers were matched based on retention time, mass assignment, and online database query. The accurate mass and structure information of the markers obtained from KEGG (www.genome.jp/kegg/), HMDB (www.hmdb.ca/), and ChemSpider (www.chemspider.com/) databases.

\subsection{Quantitation of BA Concentrations in Serum}

Serum BA concentrations were determined using the slightly modified method [16]. In brief, serum samples (50 $\mu \mathrm{L}$ ) were mixed with $50 \mu \mathrm{L}$ methanol and $50 \mu \mathrm{L}$ of isotopic standards mixture $(2000 \mathrm{ng} / \mathrm{mL})$ in an ice-cold water bath. After being mixed thoroughly for $1 \mathrm{~min}$, the mixture stood at $-20^{\circ} \mathrm{C}$ for $20 \mathrm{~min}$. The mixture was centrifuged at $16000 \times \mathrm{g}$ for $10 \mathrm{~min}$ at $4^{\circ} \mathrm{C}$, and then $2 \mu \mathrm{L}$ of the supernatant was injected to the UPLC-TOF/MS system. The UPLC system was coupled online to a Xevo Q-TOF mass spectrometer (Waters) in negative electrospray mode with a scan range of $\mathrm{m} / \mathrm{z} 50$ to 1000 . The chromatographic separation of the analytes was performed using an Acquity UPLC BEH C18 column $(100 \times 2.1 \mathrm{~mm}$ i.d., $1.7 \mu \mathrm{m}$ particles, Waters $)$. Details of the LC and MS conditions including gradient elution and the selected reaction monitoring transitions for all the compounds as well as the corresponding internal standard for each BA were described in a previous report [16]. The calibration curve was linear for all BAs over a range of 10 to $25 \mathrm{ng} / \mathrm{mL}$ (Min.) 1000 to $10000 \mathrm{ng} / \mathrm{mL}$ (Max.).

\subsection{Serum TH Concentrations}

Serum $T_{4}$ and $T_{3}$ concentrations were determined by the method using online solid-phase extraction liquid chromatography-mass spectrometry/mass spectrometry (online SPE LC-MS/MS) [17]. In brief, an API5000 triple-quadrupole mass spectrometer (AB SCIEX, CA, U.S.A.) equipped with a TurboIonSpray source and Shimadzu HPLC system was employed to perform the analysis using isotope dilution with deuterium labeled internal standard, $\left[{ }^{2} \mathrm{H}_{5}\right] \mathrm{T}_{4}$. A $20 \mu \mathrm{L}$ of rat serum was mixed with internal standard acetonitrile solution for deproteinization, and was diluted with $0.1 \%$ formic acid. After centrifugation, the supernatant was injected into the LC-MS/MS system. Online extraction and chromatographic separation of the analytes were performed using respectively a Shim pack MAYI-ODS column $(10 \mathrm{~mm} \times 2.0 \mathrm{~mm}$ i.d., $50 \mu \mathrm{m}$ particles, Shimadzu, Kyoto, Japan) and a Synergi Polar-RP 80A column (50 mm $\times$ $2.0 \mathrm{~mm}$ i.d., $4 \mu \mathrm{m}$ particles, Phenomenex, Utrecht, the Netherlands). Details of the LC conditions including gradient elution and the selected reaction monitoring transitions are described in a previous report [17]. Linear calibration curves of $\mathrm{T}_{4}$ and $\mathrm{T}_{3}$ were obtained in the concentration range of 0.1 to $100 \mathrm{ng} / \mathrm{mL}$, with a lower limit of quantitation of $0.1 \mathrm{ng} / \mathrm{mL}$. 


\subsection{Serum TSH Concentrations}

Serum TSH concentrations were determined by Enzyme-Linked Immunosorbent Assay using TSH ELISA Test Kit (Endocrine Technology, Newark, CA, U.S.A.).

\subsection{Calculations and Statistical Analysis}

Total bile acid (total BA) concentration was calculated as the sum of the 10 bile acids: CA, CDCA, GCA, GCDCA, TCA, TCDCA, TDCA, THDCA, TUDCA, and BTMCA. TLCA and THCA were not included as only trace amounts were detected. All data of serum BA concentrations were expressed as mean \pm standard deviation (S.D.). Statistical significance of serum BA concentrations between control and Tx group in the experiment 1 was determined by an unpaired Student's $t$-test. Differences between three groups in the experiment 2 were assessed by one way analysis of variance. Post hoc testing was performed for inter-group comparisons using the Tukey's test. In all cases, the results were considered significant at $p<0.05$.

\section{Results}

\subsection{Serum Metabolomic Profiling}

Serum samples were collected on the final day (3 weeks after $\mathrm{Tx}$ ) of the experiment 1 , and analyzed using UPLC-QTOF/MS. As more metabolites were detected in negative ion mode than in positive ion mode, negative ion mode was used for this study. Representative base peak ion (BPI) chromatograms of the serum of control and Tx rats are presented in Fig. 1. The variations in $m / z$ values and retention times of selected peaks were less than $5 \mathrm{mDa}$ and $0.05 \mathrm{~min}$, respectively. After peak alignment, the serum datasets contained 4456 metabolite ions. The pre-processed UPLC-QTOF/MS data were further investigated using multivariate statistical analysis.
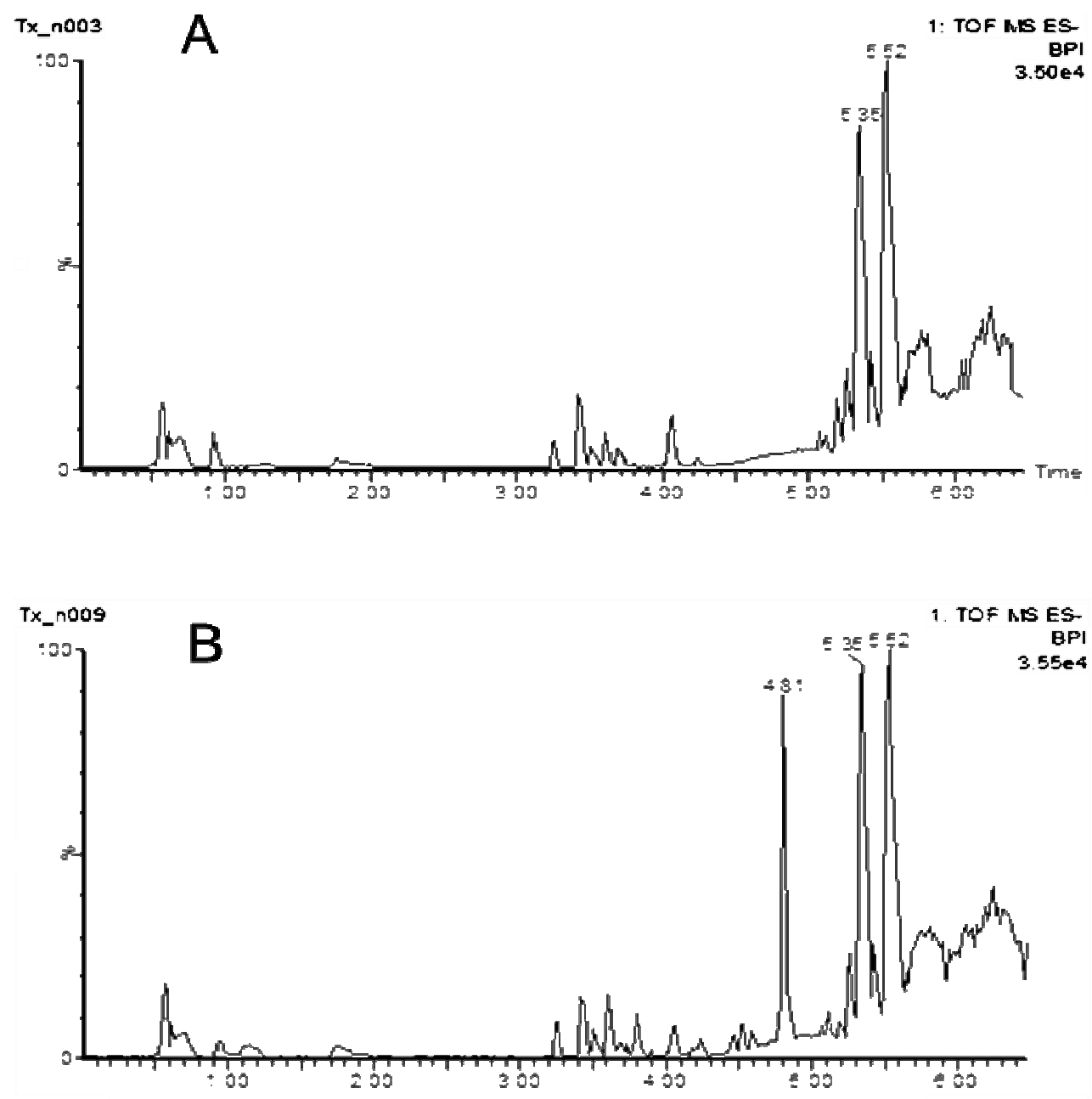

Fig. 1. The UPLC-QTOF/MS BPI profile of serum samples in (A) control group and (B) Tx group.

\subsection{Multivariate Data Analysis}

The supervised OPLS-DA was applied to obtain better discrimination between the control and Tx groups. Good separation between the two groups was observed, and there was no variation among the rats within each group and no outliers on the vertical axis (Fig. 2A). The goodness-of-fit parameter $R^{2}$ and the predictive ability parameter $Q^{2}$ were 0.999 and 0.895 , respectively. These results indicate that this 
model fits and is useful for discriminating between the two groups.

The S plots from the OPLS-DA model were created using the loading profile of the first component $(p)$ and the correlation of $p$ with the first component ( $p$ (corr)), which represents the reliability of $p$ to the first component (Fig. 2B). Each plot represents one chromatogram of a metabolite with a specific retention time. We used cut-off values of $p \geq|0.05|$ and $p$ (corr) $\geq|0.8|$, and 38 plots located inside the squares in Fig. 2B were selected; 28 plots were more increased in the Tx group compared with the control group, and 10 plots were more decreased in the Tx group compared with the control group. According to an identity check based on raw data and features of the chromatographic peaks, the candidate metabolites detected in the profiling process were identified by MS/MS analysis or by searching their accurate masses using the online metabolite databases, KEGG, HMDB, and ChemSpider. Several metabolites of pyrimidine, phenylalanine, and the amino acid metabolism pathway were matched (data not shown). Many BAs and their metabolites, CA, CDCA, GCA, GCDCA, CA glucuronide, and CDCA sulfate, were matched as markers (Table 1). Among these, the metabolite that suggested the largest change in Fig. 2B (retention time 4.79) was identified as CA.

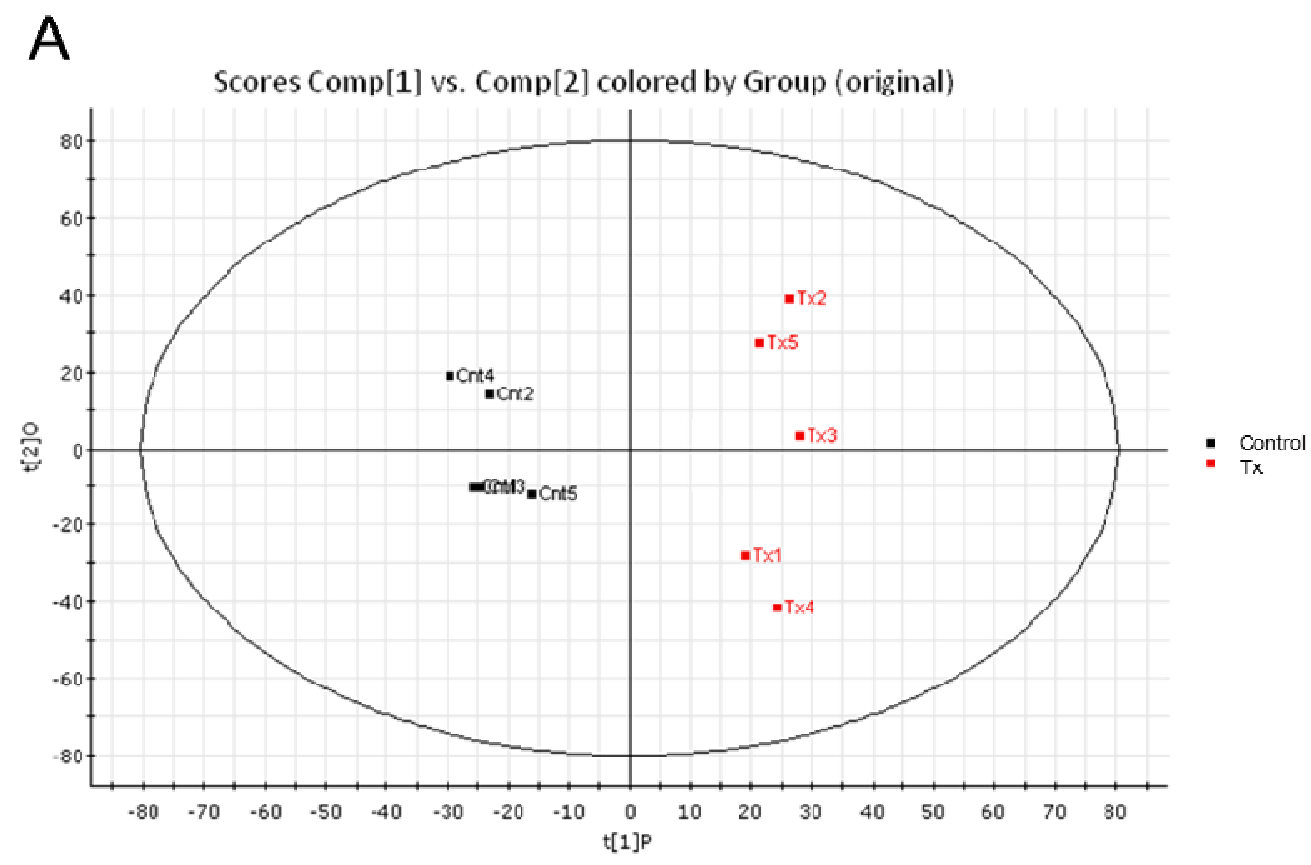

B

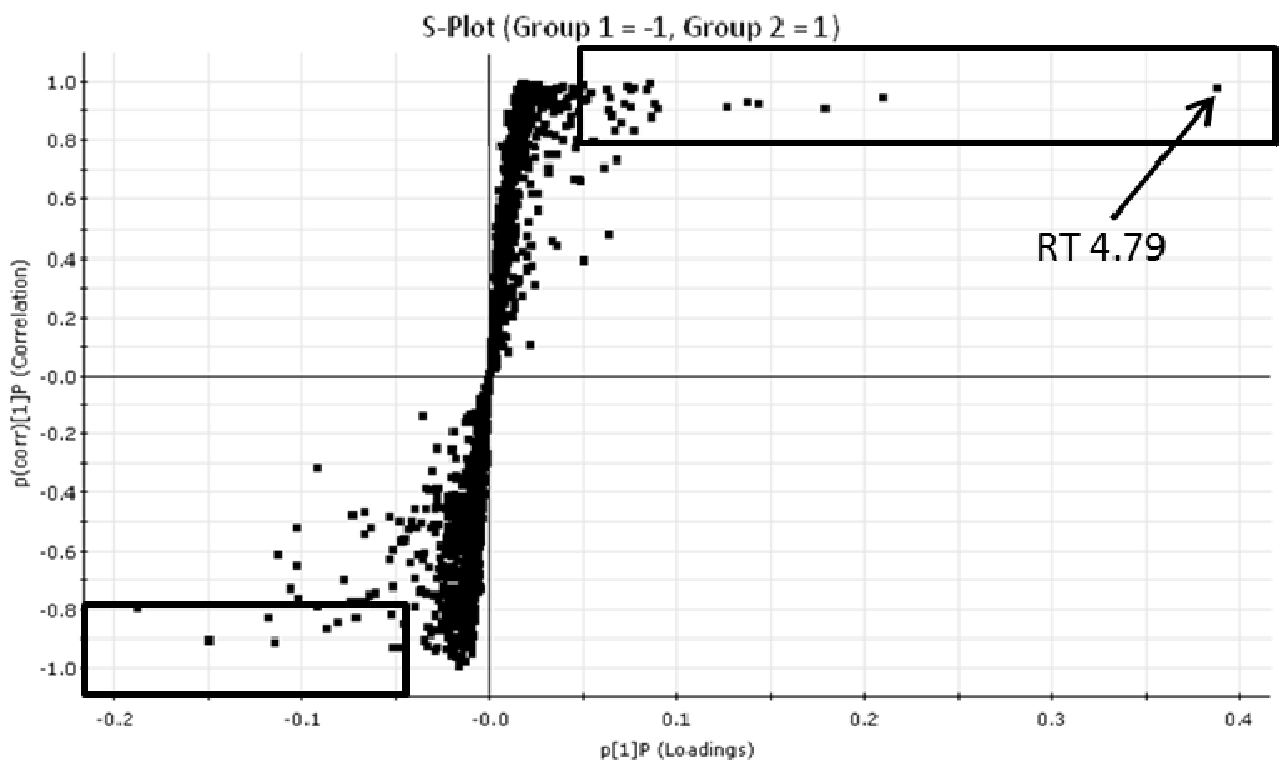

Fig. 2. OPLS-DA plots of serum samples in control group and Tx group. (A) scores plot, (B) loadings S-plot. 
Table 1. List of bile acid and their metabolites searched by online metabolite database.

\begin{tabular}{lllll}
\hline Retention time $(\mathbf{m i n})$ & Mass $(\boldsymbol{m} / \mathbf{z})$ & Metabolite & Mass accuracy $(\mathbf{m D a})^{\text {Change }^{\mathbf{a}}}$ \\
\hline 4.79 & 407.2784 & Cholic acid & -1.3 & $\uparrow$ \\
4.84 & 391.2841 & Chenodeoxycholic acid & -0.7 & $\uparrow$ \\
4.47 & 464.3010 & Glycocholic acid & 0.1 & $\uparrow$ \\
4.46 & 448.3052 & Glycochenodeoxycholic acid & 1.0 & $\uparrow$ \\
4.38 & 583.3093 & Cholic acid glucuronide & 2.5 & $\downarrow$ \\
4.50 & 471.2421 & Chenodeoxycholic acid sulfate & -0.5 & $\downarrow$ \\
\hline
\end{tabular}

${ }^{a}$ Relative level in serum of thyroidectomized group in comparison to the control group

\subsection{Changes in Serum BA Concentrations After Tx}

Metabolomic analysis using UPLC-QTOF/MS revealed that serum BAs, and especially CA, levels markedly elevated in the Tx group compared with controls at the endpoint of the experiment. Serum concentrations of primary BAs including CA, CDCA, GCA, and GCDCA were quantified using a simultaneous analytical method [16]. Sequential changes in individual serum BA levels after Tx were also analyzed.

Serum CA, CDCA, and TCDCA levels increased rapidly more 7 days after Tx, and significantly increased to 25 -fold, 11-fold, and 3-fold of the control group, respectively, 21 days after Tx (Fig. 3). CA and CDCA constituted approximately
$80 \%$ of total BA content (CA: $62 \%$ and CDCA: $18 \%$ ). Serum TDCA levels increased immediately after $\mathrm{Tx}$, and significantly increased to 21-fold the level of controls on day 21after Tx. THDCA levels did not change until 14 days after Tx, but significantly increased to 21-fold of controls on day 21 . Serum GCA, GCDCA, and TCA levels were also increased in Tx rats (2-fold, 3-fold, and 2-fold, respectively, of controls on day 21), but these differences were not statistically significant. Serum TUDCA and $\beta$ TMCA levels did not significantly differ between the control and Tx groups. Serum TLCA and THCA were not analyzed as only trace amounts were detected.
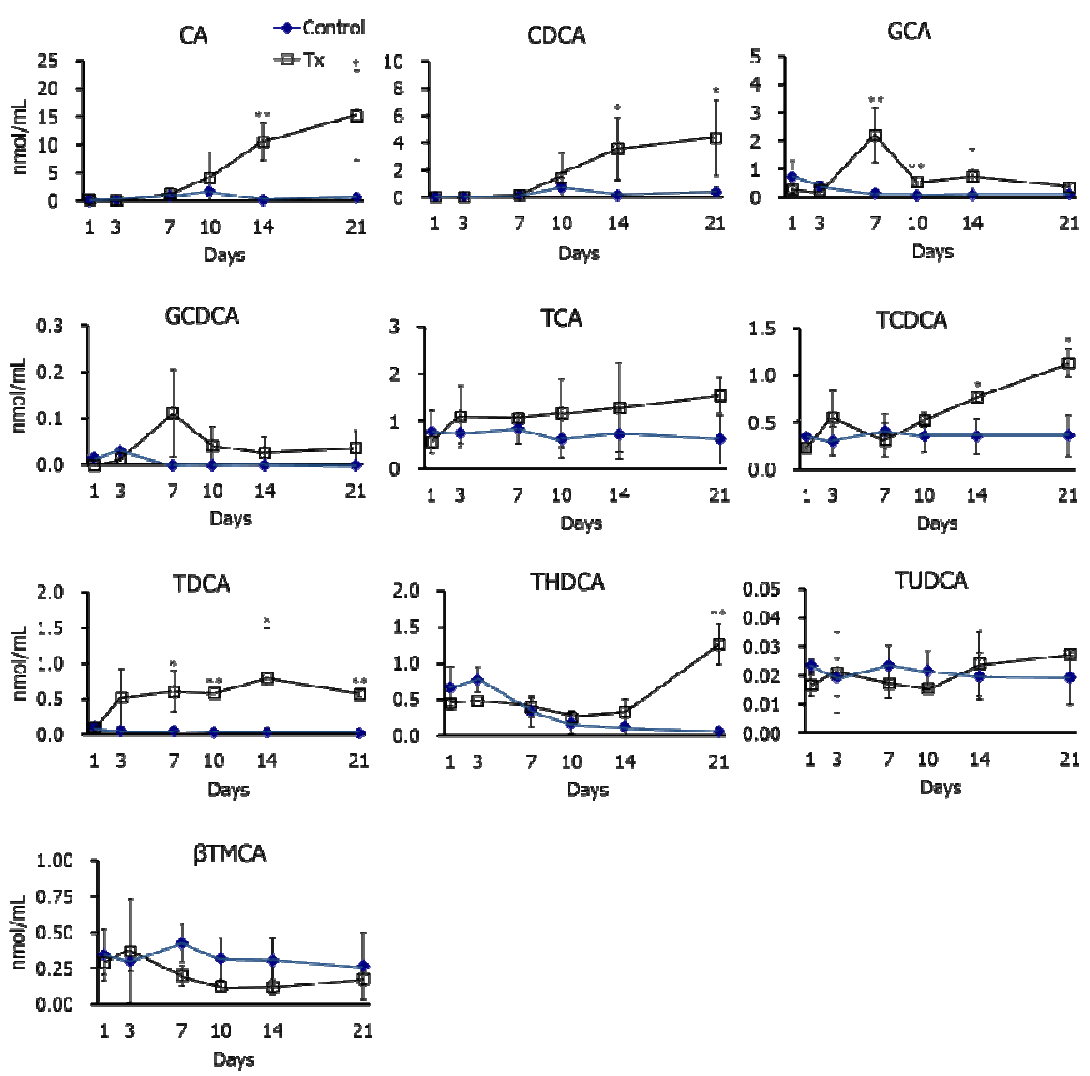

Fig. 3. Sequential changes in individual serum BA concentrations of control and Tx rats.

All data represent the mean \pm S.D. of five animals. ${ }^{*} \mathrm{p}<0.05$ and $* * \mathrm{p}<0.01$ compared with the controls. Undefined abbreviations are: CA, cholic acid; CD, chenodeoxy; G, glycol; T, tauro; D, deoxy; H, hyo; HD, hyodeoxy; UD, ursodeoxy; M, muri.

\subsection{Serum Concentrations of $\mathrm{TH}$}

Serum $\mathrm{T}_{3}$ and $\mathrm{T}_{4}$ concentrations were determined before (day -1) and after (day 14) subcutaneous implantation of osmotic minipumps, delivering vehicle (Control and Tx), 1.5 $\mu \mathrm{g} \mathrm{T}_{3} /$ head/day $(\mathrm{T} x+\mathrm{T} 3)$ to control or Tx rats (Fig. 4). In the 
all Tx groups before $T_{3}$ replacement, serum $T_{4}$ and $T_{3}$ levels significantly decreased compared with controls $\left(\mathrm{T}_{4}: 39.0 \pm 5.0\right.$ $\mathrm{ng} / \mathrm{mL}$ for control, $2.4 \pm 0.9 \mathrm{ng} / \mathrm{mL}$ for $\mathrm{Tx}, 2.6 \pm 0.6 \mathrm{ng} / \mathrm{mL}$ for $\mathrm{Tx}+\mathrm{T} 3$ and $\mathrm{T}_{3}: 0.61 \pm 13 \mathrm{ng} / \mathrm{mL}$ for control, $0.10 \pm 0.06$ $\mathrm{ng} / \mathrm{mL}$ for $\mathrm{Tx}, 0.10 \pm 0.05 \mathrm{ng} / \mathrm{mL}$ for $\mathrm{Tx}+\mathrm{T} 3$ ). These levels in Tx group did not significantly change between before and after vehicle infusion. In the $\mathrm{Tx}+\mathrm{T} 3$ group after $\mathrm{T}_{3}$ replacement, serum $T_{3}$ levels significantly increased compared with that of before replacement, and were approximately $92 \%$ of control group $(0.49 \pm 0.09 \mathrm{ng} / \mathrm{mL}$ for control, $0.45 \pm 0.06 \mathrm{ng} / \mathrm{mL}$ for $\mathrm{Tx}+\mathrm{T} 3)$.
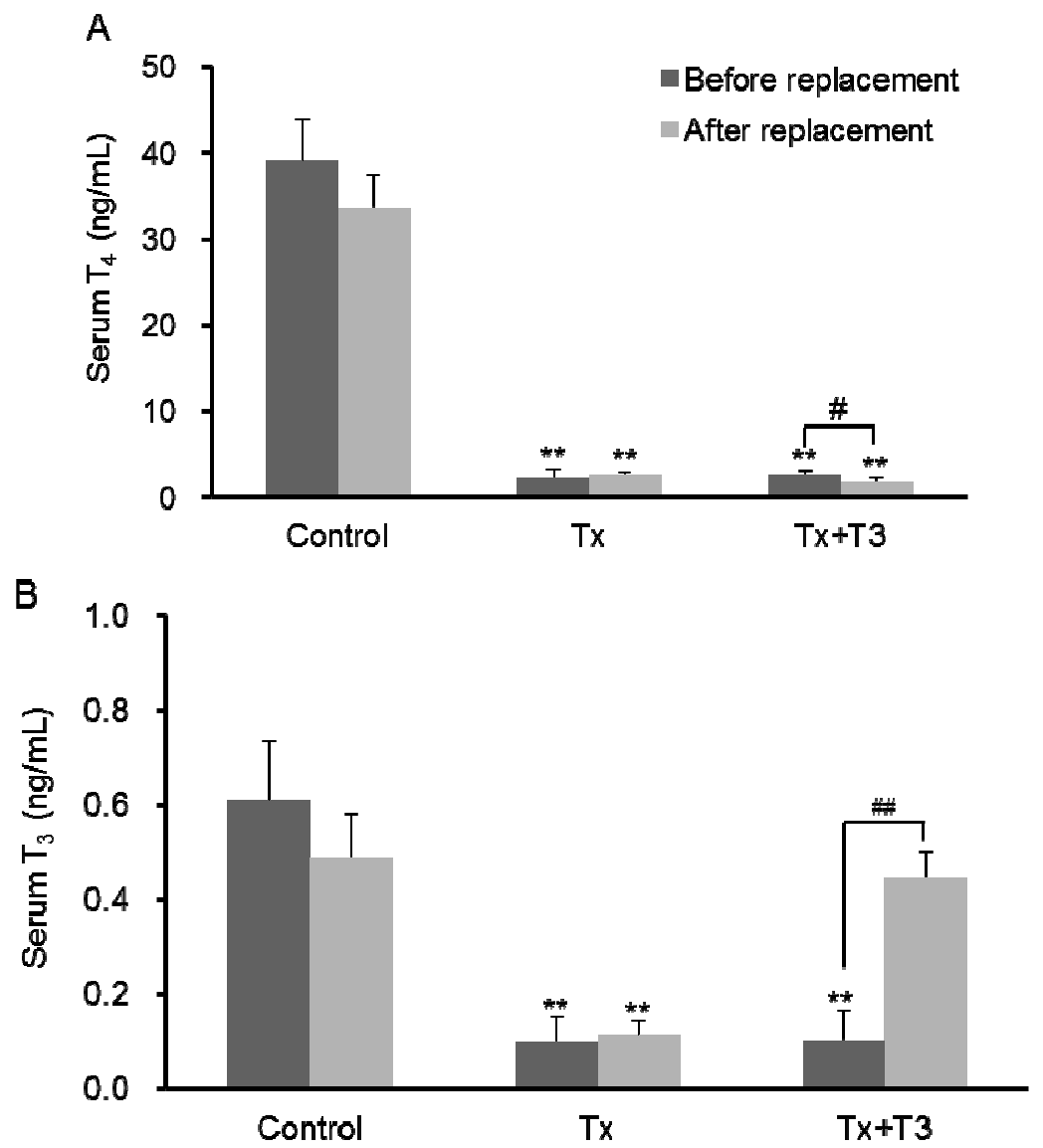

Fig. 4. Serum $T_{4}(A)$ and $T_{3}(B)$ concentrations before and after subcutaneous infusion of vehicle or $T_{3}$ in control or Tx rats.

Serum samples were collected before (day -1) and after (day 14) subcutaneous implantation of osmotic minipumps, delivering vehicle (control and Tx) or $1.5 \mu \mathrm{g}$ $\mathrm{T}_{3} /$ head/day $(\mathrm{Tx}+\mathrm{T} 3)$ to control or Tx rats. All data represent the mean \pm S.D. of six animals. ${ }^{*} p<0.05$ and $* * p<0.01$ compared with the controls. ${ }^{*} p<0.05$ and $\# p<0.01$ compared with the before replacements.

\subsection{Serum TSH Concentrations}

Table 2. Serum TSH concentrations in control and Tx rats.

\begin{tabular}{lll}
\hline \multirow{2}{*}{ Group } & Concentration $(\mathbf{n g} / \mathbf{m L})$ & After $^{\mathbf{b}}$ \\
\cline { 2 - 3 } & Before $^{\mathbf{a}}$ & $3.91 \pm 1.26^{\# \#}$ \\
Control & $1.47 \pm 0.671$ & $26.9 \pm 10.3^{\#}$ \\
$\mathrm{Tx}$ & $10.8 \pm 3.26^{* *}$ & $16.7 \pm 6.50$ \\
$\mathrm{Tx}+\mathrm{T} 3$ & $11.8 \pm 1.03^{* *}$ & $1.7 \pm$ \\
\hline
\end{tabular}

The control and Tx groups were subcutaneous implanted osmotic minipumps, delivering vehicle. The Tx+T3 group was subcutaneous implanted osmotic minipumps, delivering $\mathrm{T}_{3}\left(1.5 \mu \mathrm{g} \mathrm{T}_{3} /\right.$ head/day).

Data are expressed as the mean values \pm S.D. $(\mathrm{n}=6)$.

$* p<0.05$ and $* * p<0.01$ compared with the controls.

${ }^{\#} p<0.05$ and ${ }^{\# \#} p<0.01$ compared with the before replacements.

${ }^{\mathrm{a}}$, the day before replacement; ${ }^{\mathrm{b}}, 14$ th day after replacement.

Serum TSH concentrations were determined before (day -1) and after (day 14) subcutaneous implantation of osmotic minipumps, delivering vehicle (Control and Tx), $1.5 \mu \mathrm{g}$
$\mathrm{T}_{3} /$ head/day $(\mathrm{Tx}+\mathrm{T} 3)$ to control or $\mathrm{Tx}$ rats (Table 2$)$. In the all Tx groups before $T_{3}$ replacement, serum TSH levels significantly increased compared with controls. In the Tx 
group after vehicle infusion, serum TSH levels significantly elevated more than those of before infusion. However, in the $\mathrm{T}+\mathrm{T} 3$ group, serum TSH levels did not significantly change between before and after $\mathrm{T}_{3}$ replacement. Serum TSH levels in Tx rats did not return to normal by $\mathrm{T}_{3}$ replacement.

\subsection{Effects of $T_{3}$ Replacement on Serum BA Concentrations in Tx Rats}

To investigate the effects of $\mathrm{T}_{3}$ replacement on serum BA concentrations in Tx rats, serum total BA concentrations were determined before (day -1) and after (day 14) subcutaneous implantation of osmotic minipumps, delivering vehicle (control and Tx groups) or $1.5 \mu \mathrm{g} \mathrm{T}_{3} /$ head/day ( $\mathrm{Tx}+\mathrm{T} 3$ group) to normal or Tx rats (Table 3 ). Serum total BA concentrations in the $\mathrm{Tx}$ and $\mathrm{Tx}+\mathrm{T} 3$ groups before replacement were significantly increased compared with those of the control group (Tx: $25.3 \pm 14.8 \mathrm{nmol} / \mathrm{mL}, \mathrm{Tx}+\mathrm{T} 3: 20.7 \pm 10.7$ nmol/mL, control: $1.55 \pm 0.365 \mathrm{nmol} / \mathrm{mL}$ ). However, Serum total BA concentrations in Tx rats did not change after $\mathrm{T}_{3}$ replacement. Next, the composition ratio of each BA in serum was investigated before and after $\mathrm{T}_{3}$ replacement in Tx rats (Fig. 5). Although the composition ratios of serum GCA and GCDCA in the $\mathrm{Tx}+\mathrm{T} 3$ group were significantly increased after $\mathrm{T}_{3}$ replacement (GCA: $1.2 \%$ vs. $7.5 \%$ and GCDCA: $0.03 \%$ vs. $0.77 \%$, respectively), the composition ratios of most bile acids did not change markedly (CA: $67.7 \%$ vs. $61.5 \%$, CDCA: $20.3 \%$ vs. $20.8 \%$, TCA: $6.2 \%$ vs. $5.5 \%$, TCDCA: $1.6 \%$ vs. $1.4 \%$, TDCA: $1.0 \%$ vs. $0.6 \%$, THDCA: $1.3 \%$ vs. $0.6 \%$, TUDCA: $0.04 \%$ vs. $0.13 \%$, and $\beta$ TMCA: $0.6 \%$ vs $1.2 \%$ ).

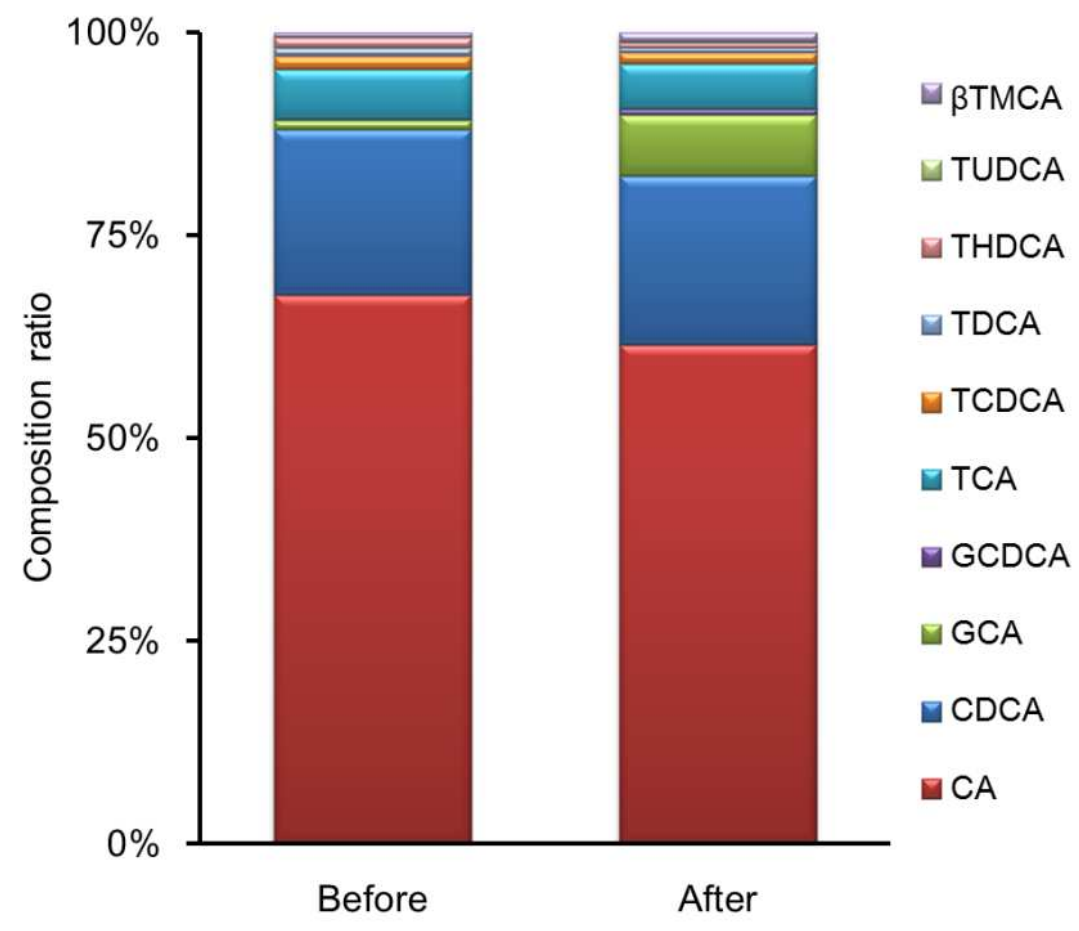

Fig. 5. Effects of $T_{3}$ replacement on serum BA composition in Tx rats.

All data represent the mean of six animals. Before, the day before replacement; After, 14th day after replacement. Undefined abbreviations are: CA, cholic acid; $\mathrm{CD}$, chenodeoxy; G, glycol; T, tauro; D, deoxy; H, hyo; HD, hyodeoxy; UD, ursodeoxy; M, muri.

Table 3. Effects of triiodothyronine replacement on serum total bile acid concentrations in Tx rats.

\begin{tabular}{lll}
\hline \multirow{2}{*}{ Group } & \multicolumn{2}{l}{ Total BA Concentration $(\mathbf{n m o l} / \mathbf{m L})$} \\
\cline { 2 - 3 } & Before $^{\mathbf{a}}$ & After $^{\mathbf{b}}$ \\
\hline Control & $1.55 \pm 0.365$ & $1.83 \pm 0.636$ \\
Tx & $25.3 \pm 14.8^{* *}$ & $32.8 \pm 20.2^{* *}$ \\
Tx+T3 & $20.7 \pm 10.7^{* *}$ & $20.4 \pm 9.27^{* *}$ \\
\hline
\end{tabular}

The control and Tx groups were subcutaneous implanted osmotic minipumps, delivering vehicle. The $\mathrm{Tx}+\mathrm{T} 3$ group was subcutaneous implanted osmotic minipumps, delivering $\mathrm{T}_{3}\left(1.5 \mu \mathrm{g} \mathrm{T}_{3} /\right.$ head/day).

Data are expressed as the mean values \pm S.D. $(n=6)$.

** $p<0.01$ compared with the controls

${ }^{\mathrm{a}}$, the day before replacement; ${ }^{\mathrm{b}}, 14$ th day after replacement.

\section{Discussion}

Hypothyroidism is caused by thyroid gland disorders, and leads to a decrease in production and secretion of $T_{4}$ and $T_{3}$. Tx rats are a well-established hypothyroidism model. In a previous study, we reported that $\mathrm{Tx}$ induced a prolongation of the half-life of $\mathrm{T}_{4}$ in serum, and influenced $\mathrm{T}_{4}$ metabolism and elimination [17]. We speculated that many endogenous metabolites were altered by $\mathrm{Tx}$.

In the present study, we performed metabolomics analysis of serum to comprehensively analyze changes in several metabolic pathways in Tx rats and to identify corresponding biochemical perturbations. We could detect more than 4000 metabolite ions in each rat, providing a very comprehensive 
serum metabolome for Tx. Using OPLS-DA followed by an online metabolite database search, metabolites that differed in Tx rats were matched, including $\mathrm{CA}, \mathrm{CDCA}, \mathrm{GCA}$, and GCDCA, which were all increased in the serum of the Tx group. These results suggest that BAs are candidate markers for hypothyroidism.

Metabolomics is very useful for biomarker investigation, but it is a qualitative analytical method. Thus, we also measured serum concentrations of 12 BAs including CA, CDCA, GCA, and GCDCA, and investigated sequential changes in individual serum BA levels after Tx. Serum concentrations of all BAs except TLCA, THCA, TUDCA, and $\beta$ TMCA, increased after Tx. There was a difference in sequential changes of each BA after Tx. BAs are synthesized from cholesterol in the liver. There are many steps, and multiple intermediates involved in the formation of primary BAs, which are CA and CDCA. These BAs were dehydroxylated by intestinal bacteria to form secondary BAs deoxycholic acid and lithocholic acid. Moreover, they undergo amidation with taurine or glycine to form conjugated BAs. Thus, we considered that the difference in sequential changes of each BA after Tx would be caused by the metabolic process of BA. Among these, serum concentrations of CA, CDCA, and TCDCA were significantly increased than 7 days after Tx. In a previous study, we have demonstrated that serum concentrations of $\mathrm{T}_{4}$ and $T_{3}$ decrease rapidly after $T x$, and are maintained at very low levels than 7 days after treatment [17]. Thus, these results suggest that the increased CA, CDCA, and TCDCA levels in serum after Tx are a homeostatic response to serum thyroid hormone deficiency.

Although BAs have been known to play an essential role indietary lipid absorption and cholesterol metabolism, in recent years BAs have been shown to act as signaling molecules through activation of a variety of nuclear and cell surface receptors [20]. Membrane-type bile acid receptor (M-BAR)/TGR5 is a member of the G-protein coupled receptor (GPCR) superfamily, and has been found to be ubiquitously expressed including in endocrine glands, brown adipose tissue (BAT), muscles, immune organs, and the enteric nervous system in humans and animals [21]. TGR5 induces the production of intracellular cyclic adenosine monophosphate (cAMP) upon interaction with BAs [21,22]. Watanabe et al. reported that deiodinase type 2 (D2) is activated by increased cAMP production that stems from the binding of BAs with TGR5 in human brown adipocytes and skeletal myocytes, and then activated D2 accelerates to convert the pro-hormone $\mathrm{T}_{4}$ to active hormone $\mathrm{T}_{3}$, a major component involved in cellular basal metabolism [23]. Intracellular $\mathrm{T}_{4}$ and $\mathrm{T}_{3}$ levels decrease after $\mathrm{Tx}$ [24]. Moreover, we previously reported that activity and mRNA expression of D2 in brain, BAT, and muscle were increased after Tx in rats [25]. Thus, our findings suggest that the BAs (CA, CDCA, and TCDCA) act as a signaling molecule to increase intracellular $\mathrm{T}_{3}$ levels in local tissues by accelerating the above mechanisms (the BA-TGR5-cAMP-D2- T $_{3}$ pathway).

In hypothyroid patients, $\mathrm{T}_{4}$ and $\mathrm{T}_{3}$ levels in both blood and tissues are markedly decreased but return to physiological levels with $\mathrm{T}_{4}$ replacement therapy [26]. $\mathrm{T}_{4}$ is considered a pro-hormone, as activation occurs through deiodination of $\mathrm{T}_{4}$ to form $\mathrm{T}_{3}$, the active form of $\mathrm{TH}$. Thus, $\mathrm{T}_{3}$ replacement therapy in hypothyroid patients may be more effective than $T_{4}$ replacement. In this study, we investigated the effects of $T_{3}$ replacement on serum BA concentrations in hypothyroid rats. Serum $\mathrm{T}_{3}$ levels in $\mathrm{Tx}$ rats were returned to physiological levels with $\mathrm{T}_{3}$ replacement. However, serum TSH levels were maintained at very high levels. These results were consistent with a previously study by Escobar-Morrealeet al.[27]. They also reported that $T_{3}$ levels in many tissues of $T x$ rats were distinctly lower than the controls even if serum $\mathrm{T}_{3}$ levels were returned to physiological levels with $\mathrm{T}_{3}$ replacement. Serum concentrations of total $\mathrm{BA}$ in Tx rats after $\mathrm{T}_{3}$ replacement showed little change compared with before replacement, and the composition ratio of BAs also showed no significant difference before and after $T_{3}$ replacement. Intracellular $T_{3}$ levels are known to be maintained by direct $\mathrm{T}_{3}$ uptake from blood and by intracellular selective conversion of $\mathrm{T}_{4}$ to $\mathrm{T}_{3}$ by D2 [28,29]. Intracellular $\mathrm{T}_{3}$ concentrations in BAT and brain are supplied at a ratio of 50:50 and 20:80 from these two pathways, respectively $[30,31]$. Moreover, we have previously reported that activity and mRNA expression of D2 in local tissues of Tx rats were maintained at high levels after $\mathrm{T}_{3}$ replacement [25]. These results indicate that serum BA levels were maintained at a high level because intracellular $\mathrm{TH}$ concentrations in $\mathrm{Tx}$ rats were not normalized to physiological levels by $\mathrm{T}_{3}$ replacement.

$\mathrm{TH}$ replacement therapy in hypothyroid patients is estimated by serum levels of THs and TSH. In general, TH in the blood is quickly taken up into cells in target tissues via specific plasma membrane transporters, members of the organic anion transporting polypeptide family [32], and the monocarboxylate transporter family [33]. However, in humans and rodents who exhibit inactivating mutations in the genes encoding these transporters, THs in the blood cannot be taken up into cells even after TH replacement, and biological function is not fully restored [34-36]. Thus, we suggest that $\mathrm{TH}$ levels in local tissues area more important indicator of the efficacy of hypothyroidism therapy than TH levels in blood. However, it is unfeasible to measure $\mathrm{TH}$ concentrations in human patients' organ. The results of study indicate that serum BA levels are negatively correlated with intracellular TH concentrations in target tissues. Thus, serum CA, CDCA, and TCDCA may be potential biomarkers for hypothyroidism and the efficacy of TH replacement therapy in hypothyroid patients. To identify this hypothesis, further studies are needed to investigate the mechanism by which Tx increases serum BA levels in rats, and to determine if the same results can be seen in human subjects.

\section{Conclusion}

Serum BA levels were shown to increase markedly in rats after $\mathrm{Tx}$, as indicated by metabolomics analysis. Moreover, we investigated sequential changes in serum levels of 12 BAs 
in rats after Tx. We observed an increase in serum CA, CDCA, and TCDCA after Tx, and believe that this is a homeostatic response to intracellular TH deficiency in local tissues. To the best of our knowledge, this is the first report indicating that serum CA, CDCA, and TCDCA may be potential biomarkers for hypothyroidism and the efficacy of $\mathrm{TH}$ replacement therapy in hypothyroidism.

\section{Acknowledgements}

We thank to Ms. Etsuko Suzuki and Mr. Kazunobu Maruyama (Waters) for technical assistance.

\section{References}

[1] T.J. Visser, "Pathways of thyroid hormone metabolism," Acta Med. Austriaca, vol. 23, pp. 10-16, 1996.

[2] S.Y. Cheng, "Multiple mechanisms for regulation of the transcriptional activity of thyroid hormone receptors," Rev. Endocr. Metab. Disord., vol. 1, pp. 9-18, 2000.

[3] G.R. Williams, "Cloning and characterization of two novel thyroid hormone receptor $\beta$ isoforms," Mol. Cell. Biol., vol. 20, pp. 8329-8342, 2000.

[4] H. Ying, H. Suzuki, L. Zhao, M.C. Willingham, P. Meltzer and S.Y. Cheng, "Mutant thyroid hormone receptor $\beta$ represses the expression and transcriptional activity of peroxisome proliferator-activated receptor $\gamma$ during thyroid carcinogenesis," Cancer Res., vol. 63, pp. 5274-5280, 2003.

[5] I. Jones, L. Ng, H. Liu and D. Forrest, "An intron control region differentially regulates expression of thyroid hormone receptor $\beta 2$ in the cochlea, pituitary, and cone photoreceptors," Mol. Endocrinol., vol. 21, pp. 1108-1119, 2007.

[6] A.R. Cappola and P.W. Ladenson, "Hypothyroidism and atherosclerosis," J. Clin. Endocrinol. Metab., vol. 88, pp. 2438-2444, 2003.

[7] P. Cettour-Rose, C. Theander-Carrillo, C. Asensio, M. Klein, T.J. Visser, A.G. Burger, C.A. Meier and F. Rohner-Jeanrenaud, "Hypothyroidism in rats decreases peripheral glucose utilisation, a defect partially corrected by central leptin infusion," Diabetologia, vol. 48, pp. 624-633, 2005.

[8] A. Zhang, H. Sun, G. Yan, Y. Han, Y. Ye and X. Wang, "Urinary metabolic profiling identifies a key role for glycocholic acid in human liver cancer by ultra-performance liquid-chromatography coupled with high-definition mass spectrometry," Clin. Chim. Acta, vol. 418, pp. 86-90, 2013.

[9] N. Aranibar, M. Borys, N.A. Mackin, V. Ly, N. Abu-Absi, S. Abu-Absi, M. Niemitz, B. Schilling, Z.J. Li, B. Brock, R.J. Russell 2nd, A. Tymiak and M.D. Reily, "NMR-based metabolomics of mammalian cell and tissue cultures," J. Biomol. NMR, vol. 49, pp. 195-206, 2011.

[10] A. Lodi and S.M. Ronen, "Magnetic resonance spectroscopy detectable metabolomics fingerprint of response to antineoplastic treatment," PLoS ONE, 6:e26155, 2011.

[11] L.M. Raamsdonk, B. Teusink, D. Broadhurst, N.S. Zhang, A. Hayes, M.C. Walsh, J.A. Berden, K.M. Brindle, D.B. Kell, J.J. Rowland, H.V. Westerhoff, K. van Dam and S.G. Oliver, "A functional genomics strategy that uses metabolome data to reveal the phenotype of silent mutations," Nat. Biotechnol., vol. 19, pp. 45-50, 2001.

[12] O. Fiehn, J. Kopka, P. Dormann, T. Altmann, R.N. Trethewey and L. Willmitzer, "Metabolite profiling for plant functional genomics,” Nat. Biotechnol., vol. 18, pp. 1157-1161, 2000.

[13] L.D. Roberts, A. Koulman and J.L. Griffin, "Towards metabolic biomarkers of insulin resistance and type 2 diabetes: progress from the metabolome," Lancet Diabetes Endocrinol., vol. 2, pp. 65-75, 2014.

[14] T. Soga, M. Sugimoto, M. Honma, M. Mori, K. Igarashi, K. Kashikura, S. Ikeda, A. Hirayama, T. Yamamoto, H. Yoshida, M. Otsuka, S. Tsuji, Y. Yatomi, T. Sakuragawa, H. Watanabe, K. Nihei, T. Saito, S. Kawata, H. Suzuki, M. Tomita and M. Suematsu, "Serum metabolomics reveals $\gamma$-glutamyl dipeptides as biomarkers for discrimination among different forms of liver disease,” J. Hepatol., vol. 55, pp. 896-905, 2011.

[15] T.H. Kim, M.Y. Ahn, H.J. Lim, Y.J. Lee, Y.J. Shin, U. De, J. Lee, B.M. Lee, S. Kim and H.S. Kim, "Evaluation of metabolomic profiling against renal toxicity in Sprague-Dawley rats treated with melamine and cyanuric acid," Arch. Toxicol., vol. 86, pp. 1885-1897, 2012.

[16] E.J. Want, M. Coen, P. Masson, H.C. Keun, J.T. Pearce, M.D. Reily, D.G. Robertson, C.M. Rohde, E. Holmes, J.C. Lindon, R.S. Plumb and J.K. Nicholson, "Ultra performance liquid chromatography-mass spectrometry profiling of bile acid metabolites in biofluids: application to experimental toxicology studies," Anal. Chem., vol. 82, pp. 5282-8289, 2010.

[17] H. Nagao, T. Imazu, H. Hayashi, K. Takahashi and K. Minato, "Influence of thyroidectomy on thyroxine metabolism and turnoverrate in rats," J. Endocrinol., vol. 210, pp. 117-123, 2011.

[18] J.T. Herlihy, C. Stacy and H.A. Bertrand, "Long-term food restriction depresses serum thyroid hormone concentrations in the rat," Mech. Ageing Dev., vol. 53, pp. 9-16, 1990.

[19] T.T. Nguyen, J.J. Di Stefano, H. Yamada and Y.M. Yen, "Steady state organ distribution and metabolism of thyroxine and 3,5,3'-triiodothyronine in intestine, liver, kidneys, blood and residual carcass of the rat in vivo," Endocrinology, vol. 133, pp. 2973-2983, 1993.

[20] S. Fiorucci, S. Cipriani, F. Baldelli and A. Mencarelli, "Bile acid-activated receptors in the treatment of dyslipidemia and related disorders," Prog. Lipid Res., vol. 49, pp. 171-185, 2010.

[21] Y. Kawamata, R. Fujii, M. Hosoya, M. Harada, H. Yoshida, M Miwa, S. Fukusumi, Y. Habata, T. Itoh, Y. Shintani, S. Hinuma, Y. Fujisawa and M. Fujino, "A G protein-coupled receptor responsive to bile acids," J. Biol. Chem., vol. 278, pp. 9435-9440, 2003.

[22] T. Maruyama, Y. Miyamoto, T. Nakamura, Y. Tamai, H. Okada, E. Sugiyama, T. Nakamura, H. Itadani and K. Tanaka, "Identification of membrane-type receptor for bile acids (M-BAR)," Biochem. Biophys. Res. Commun., vol. 298, pp. 714-719, 2002.

[23] M. Watanabe, S.M. Houten, C. Mataki, M.A. Christoffolete, B.W. Kim, H. Sato, N. Messaddeq, J.W. Harney, O. Ezaki, T. Kodama, K. Schoonjans, A.C. Bianco and J. Auwerx, "Bile acids induce energy expenditure by promoting intracellular thyroid hormone activation," Nature, vol. 439, pp. 484-489, 2006. 
[24] H.F. Escobar-Morreale, M.J. Obregon, F. Escobar Del Rey and G. Morreale De Escobar, "Replacement therapy for hypothyroidism with thyroxine alone does not ensure euthyroidism in all tissues, as studied in thyroidectomized rats," J. Clin. Invest., vol. 96, pp. 2828-2838, 1995.

[25] H. Nagao, M. Sasaki, T. Imazu, K. Takahashi, H. Aoki and K. Minato, "Effects of triiodothyronine on turnover rate and metabolizing enzymes for thyroxine in thyroidectomizedrats," Life Sci., vol. 116, pp. 74-82, 2014.

[26] A.D. Toft, “Thyroxine therapy,” N. Engl. J. Med., vol. 331, pp. 174-180, 1994.

[27] H.F. Escobar-Morreale, M.J. Obregón, F. Escobar del Rey and G. Morreale de Escobar, "Tissue-specific patterns of changes in 3,5,3'-triiodo-L-thyronine concentrations in thyroidectomized rats infused with increasing doses of the hormone. Which are the regulatory mechanisms?," Biochimie, vol. 81, pp. 453-462, 1999.

[28] G.R. Williams and J.H. Bassett, "Deiodinases: the balance of thyroid hormone: local control of thyroid hormone action: role of type 2 deiodinase," J. Endocrinol., vol. 209, pp. 261-272, 2011.

[29] A.C. Bianco, D. Salvatore, B. Gereben, M.J. Berry and P.R. Larsen, "Biochemistry, cellular and molecular biology, and physiological roles of the iodothyronine selenodeiodinases," Endocr. Rev., vol. 23, pp. 38-89, 2002.

[30] A.C. Bianco and J.E. Silva, "Nuclear 3,5,3'-triiodothyronine (T3) in brown adipose tissue: receptor occupancy and sources of T3 as determined by in vivo techniques," Endocrinology, vol. 120, pp. 55-62, 1987.
[31] J. van Doorn, F. Roelfsema and D. van der Heide, "Conversion of thyroxine to 3,5,3'-triiodothyronine in several rat tissues in vivo: the effect of hypothyroidism," Acta Endocrinol., vol. 113, pp. 59-64, 1986.

[32] B. Hagenbuch, "Cellular entry of thyroid hormones by organic anion transporting polypeptides," Best Pract. Res. Clin. Endocrinol. Metab., vol. 21, pp. 209-221, 2007.

[33] W.E. Visser, E.C. Friesema, J. Jansen and T.J. Visser, "Thyroid hormone transport by monocarboxylate transporters," Best Pract. Res. Clin. Endocrinol. Metab., vol. 21, pp. 223-236, 2007.

[34] E.C. Friesema, A. Grueters, H. Biebermann, H. Krude, A. von Moers, M. Reeser, T.G. Barrett, E.E. Mancilla, J. Svensson, M.H. Kester, G.G. Kuiper, S. Balkassmi, A.G. Uitterlinden, J. Koehrle, P. Rodien, A.P. Halestrap and T.J. Visser, "Association between mutations in a thyroid hormone transporter and severe X-linked psychomotor retardation," Lancet, vol. 364, pp. 1435-1437, 2004.

[35] L.M. Roberts, K. Woodford, M. Zhou, D.S. Black, J.E. Haggerty, E.H. Tate, K.K. Grindstaff, W. Mengesha, C. Raman and N. Zerangue, "Expression of the thyroid hormone transporters monocarboxylate transporter-8 (SLC16A2) and organic ion transporter-14 (SLCO1C1) at the blood-brain barrier," Endocrinology, vol. 149, pp. 6251-6261, 2008.

[36] S. Mayerl, T.J. Visser, V.M. Darras, S. Horn and H. Heuer, "Impact of Oatp1c1 deficiency on thyroid hormone metabolism and action in the mouse brain," Endocrinology, vol. 153, pp. 1528-1537, 2012. 